\title{
Tracking the dynamics of circulating tumour cell phenotypes using nanoparticle-mediated magnetic ranking
}

\author{
Mahla Poudineh, Peter M. Aldridge, Sharif Ahmed, Brenda J. \\ Green, Leyla Kermanshah, Vivian Nguyen, Carmen Tu, Reza M. \\ Mohamadi, Robert K. Nam, Aaron Hansen, Srikala S. Sridhar, \\ Antonio Finelli, Neil E. Fleshner, Anthony M. Joshua, Edward H. \\ Sargent, and Shana O. Kelley
}

Version Post-Print/Accepted Manuscript

Citation Poudineh, M., Aldridge, P., Ahmed, S., Green, B., Kermanshah, L., \&

(published version) Nguyen, V. et al. (2016). Tracking the dynamics of circulating tumour cell phenotypes using nanoparticle-mediated magnetic ranking. Nature Nanotechnology. http://dx.doi.org/10.1038/nnano.2016.239

Publisher's Statement The final published version is available at Nature Publishing Group via http://dx.doi.org/10.1038/nnano.2016.239.

Always cite the published version, so the author(s) will receive recognition through services that track citation counts, e.g. Scopus. If you need to cite the page number of the TSpace version (original manuscript or accepted manuscript) because you cannot access the published version, then cite the TSpace version in addition to the published version using the permanent URI (handle) found on the record page. 


\section{Tracking the Dynamics of Circulating Tumour Cell Phenotypes Using Nanoparticle-Mediated Magnetic Ranking}

Mahla Poudineh, ${ }^{1}$ Peter M. Aldridge, ${ }^{2}$ Sharif Ahmed, ${ }^{3}$ Brenda J. Green, ${ }^{2}$ Leyla Kermanshah, ${ }^{2}$ Vivian Nguyen, ${ }^{3}$ Carmen Tu, ${ }^{3}$ Reza M. Mohamedi, ${ }^{3}$ Robert K. Nam, ${ }^{4}$ Aaron Hansen, ${ }^{5}$ Srikala S. Sridhar, ${ }^{5}$ Antonio Finelli, ${ }^{5}$ Neil E. Fleshner, ${ }^{5}$ Anthony $M$. Joshua, ${ }^{5}$ Edward H. Sargent, ${ }^{1 *}$ Shana O. Kelley ${ }^{2,3,6^{*}}$

${ }^{1}$ Department of Electrical and Computer Engineering, Faculty of Engineering, University of

Toronto, Toronto, M5S 3G4, Canada. ${ }^{2}$ Institute for Biomaterials and Biomedical

Engineering, University of Toronto, Toronto, M5S 3M2, Canada. ${ }^{3}$ Department of Pharmaceutical Science, Leslie Dan Faculty of Pharmacy, University of Toronto, Toronto, M5S 3M2, Canada. ${ }^{4}$ Sunnybrook Health Sciences Centre, Sunnybrook Research Institute,

University of Toronto, Toronto, ON, M4N 3M5. ${ }^{5}$ Princess Margaret Cancer Centre, University Health Network, University of Toronto, Toronto, ON M5G 2M9. ${ }^{6}$ Department of Biochemistry, Faculty of Medicine, University of Toronto, Toronto, M5S 1A8, Canada.

‘Address correspondence to: shana.kelley@utoronto.ca, ted.sargent@utoronto.ca

Profiling the heterogeneous phenotypes of rare circulating tumour cells (CTCs) in whole blood is critical to unraveling the complex and dynamic properties of these potential clinical markers. This task is challenging because these cells are present at parts per billion levels among normal blood cells. Here, we report a new nanoparticle-enabled method for CTC characterization, called magnetic ranking cytometry, which profiles CTCs based on their surface expression phenotype. We achieve this using a microfluidic chip that successfully processes whole blood samples. The approach classifies CTCs with single-cell resolution in accordance with their expression of phenotypic surface markers, which is read out using magnetic nanoparticles. We deploy this new technique to reveal the dynamic phenotypes of CTCs in unprocessed blood from mice as a function of tumour growth and aggressiveness. We also test magnetic ranking cytometry using blood samples collected from cancer patients. 
The metastasis of cancerous tumours relies on the release of circulating cells that migrate to distant sites and form secondary tumours ${ }^{1,2}$. The factors that determine the invasiveness of these circulating tumour cells (CTCs) remain poorly defined, and it is not currently possible to distinguish CTCs having high versus low metastatic potential. Studying CTCs directly collected from unprocessed blood samples is a challenge given their rarity (parts per billion) in the bloodstream ${ }^{3,4}$. Moreover, these cells are highly heterogeneous: multiple cell phenotypes can exist within a given tumour, and their properties evolve dynamically once they leave a tumour and enter the bloodstream ${ }^{1}$.

Fluorescence-activated cell sorting (FACS) is a powerful present-day method to characterize and sort heterogeneous cell subpopulations. Unfortunately, FACS does not possess the sensitivity required to enable the routine characterization of CTCs at the levels that they are present in the bloodstream, and is therefore not broadly applicable to the analysis of rare cells in clinical specimens. Microfluidics-based approaches have provided a new avenue to study CTCs ${ }^{5-17}$; however, existing techniques are generally limited to the capture and enumeration of CTCs, and do not report on phenotypic properties of CTCs.

New methods are urgently needed to characterize and sort CTCs according to their detailed phenotypic profiles so that the properties of invasive versus noninvasive cells can be identified. High levels of sensitivity and resolution are required to generate profiles that will provide biological and clinical insights. Recently, we reported a method that allowed us to sort CTC subpopulations coarsely according to their phenotypic properties ${ }^{18}$. The resolution that was achieved, however, enabled discrimination among surface expression levels only when very large differences were at play. We hypothesized that much greater resolution would be required in order to profile accurately the phenotypes of CTCs to connect their molecular-level properties with invasiveness.

Here, we report a novel approach that exploits nanoparticle-mediated cell sorting, and relies on a unique chip architecture that achieves excellent control over an applied magnetic field along a channel. In this way, this new system accomplishes high-resolution phenotypic ranking of CTCs. We term the new 
approach, which is based on the longitudinal profile of magnetic field gradients, Magnetic Ranking Cytometry (MagRC). MagRC generates a phenotypic profile of CTCs using information collected at the single cell level. We show that it allows sorting of CTCs into one hundred different capture zones. We find that MagRC has a very high level of sensitivity and is able to profile CTCs accurately even when they are present at low levels $(10 \mathrm{cells} / \mathrm{ml})$ in unprocessed blood. The strategy allows the dynamic properties of CTCs to be tracked as a function of tumour growth and aggressiveness. We showcase herein, using blood samples both from xenografted mice and from human cancer patients, that the increased resolving power of MagRC provides distinct new information not accessible using existing methods.

\section{Overview of the magnetic ranking cytometry approach}

The MagRC approach leverages immunomagnetic separation ${ }^{19}$ for profiling CTCs as a function of their surface marker expression. A whole blood sample is incubated with antibody-functionalized magnetic nanoparticles that bind specifically to a corresponding surface marker, and microengineered structures inside the device enable the rare cell profiling capability of MagRC. ' $X$ '-shaped structures within the microfluidic channel generate regions with slow flow and favorable capture dynamics $^{18}$, a requirement for the capture of cells tagged with magnetic nanoparticles; while highly discretized sorting of subpopulations is achieved via the introduction of differently sized nickel micromagnets. The local magnetic force is engineered to vary systematically within the device via the footprint of the micromagnets (Figure 1B). The micromagnets are positioned concentrically within the ' $X$ '-microstructures, creating regions with low flow and high magnetic field gradients, ideal for capturing CTCs with even low levels of magnetic loading (Figure 1A). 

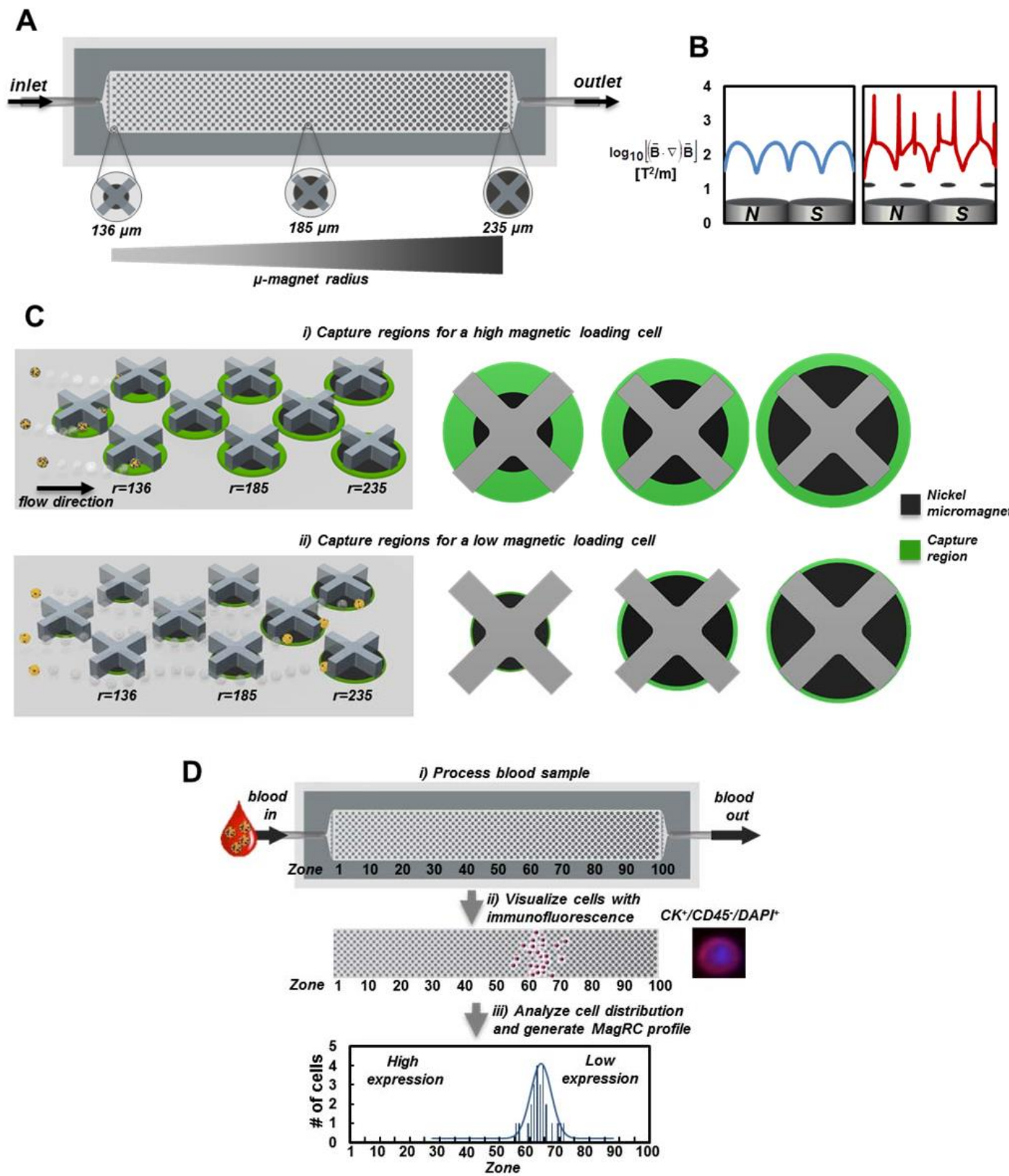

Fig. 1. The Magnetic Ranking Cytometry (MagRC) approach to profiling rare cells. A) The microfluidic chip used for MagRC contains 100 distinct zones with varied magnetic forces. An array of X-shaped structures generates regions of locally low velocity, and circular nickel micro-magnets patterned within the channel enhance the externally applied magnetic field. Increasing the size of the micromagnets along the channel increases their region of influence, where high magnetic field gradients lead to efficient CTC capture; these regions are termed capture zones. B) Comparison of the field gradient in the absence (left) and the presence (right) of $\mathrm{Ni}$ micromagnets. The micromagnets generate enhanced field gradients inside the microfluidic channel. The field gradient was measured at the channel height of $5 \mu \mathrm{m}$. C) Schematic representation of capture zones in a condensed MagRC chip. The green annuli represent capture regions where cells with varied nanoparticle loadings are predicted to be captured efficiently. CTCs with high levels of surface marker expression experience larger effective capture regions as they flow through the chip. Cells with high levels of surface marker expression (and thus high magnetic loading) are captured in the earliest zones where the micro-magnets are small ( $i$, top), while for low expression cells, the larger micro-magnets found later in the chip are required to generate a sufficiently large capture region (ii, bottom). D) Overview of the MagRC approach. i) Whole, unprocessed blood is introduced into the microfluidic chip. Once the sample has been processed, the chip is washed with buffer. Immunostaining is then used to identify CTCs and their distribution within the chip. The number of cells in each zone is then tabulated and used to generate a profile that reflects levels of protein expression for the cells as a collective. 
A quantitative physical model of the device (see supplementary material, Figure. S1 - S4) was developed to explore how cells exhibiting varied expression levels would generate different MagRC profiles that manifested their distinct phenotypes. A capture volume was defined as a region in which the magnitudes of the magnetic and drag forces are comparable. As a result, those cells that pass through a capture zone will be deflected and captured. For a cell covered with an abundance of bound magnetic nanoparticles, the capture zones generated by even the smallest micromagnets are sufficient to ensure substantially complete capture in the earliest zones of the MagRC Chip (Figure 1C, top). Cells with low surface marker expression are deflected only if they are close to edges of the micromagnets, where the magnetic force acting on the nanoparticles is highest (Figure 1C, bottom). Since each micromagnet is positioned concentrically with an ' $X$ '-structure, the regions in the MagRC chip exhibiting the highest magnetic forces and field gradients also correspond to the regions exhibiting the slowest flows. This has the benefit of creating localized regions with favorable capture dynamics (low drag and high magnetic forces), while also contributing to the high-resolution sorting capability of the chip.

For each cell in each zone, the probability of that cell encountering a capture region was calculated and reported as the capture parameter. Since the nickel micromagnets generate amplified magnetic fields near the bottom of the microfluidic channel, the capture parameter of a given cell within the chip depends strongly on its vertical position. Additionally, the extended length of the chip relative to its height leads to long residence times and the potential for cells to settle towards the bottom of the chip. The vertical dependence of the capture parameter for cells having different levels of magnetic loading is illustrated in Figure 2A. Thousands of model cells were simulated, each having a randomly assigned initial height ranging from $5 \mu \mathrm{m}$ to $45 \mu \mathrm{m}$ at the inlet of the microfluidic chip. The overall modeling results presented in Figure 2B show the predicted capture locations for three types of cells having high, medium and low levels of magnetic loading. (See supplementary material for a detailed explanation of the parametric model). 
A
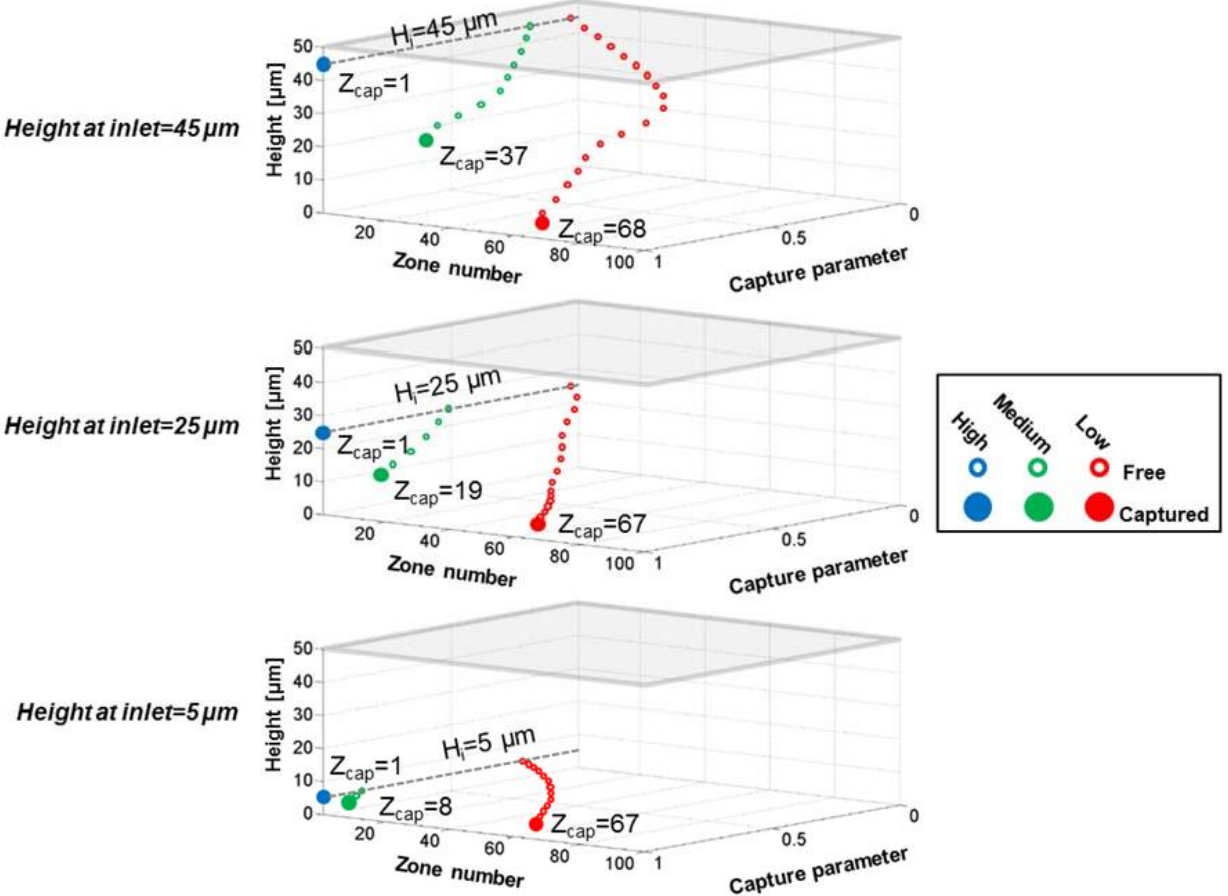

B

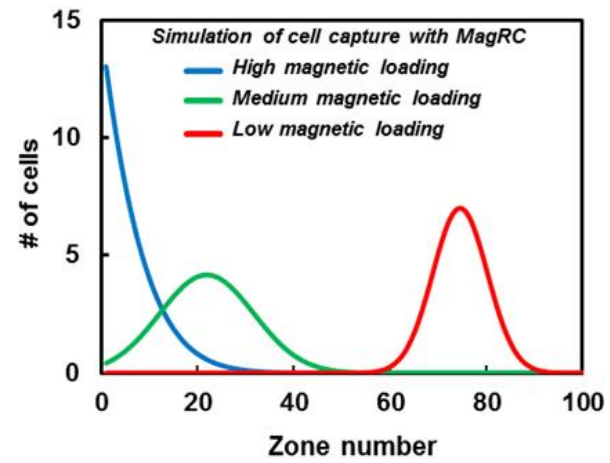

Fig 2. Modeling of cell capture in the MagRC device. A) Normalized capture parameter as a function of height and zone in the chip, for three different inlet heights. B) A parametric model predicts where high, medium and low magnetically loaded cells will be captured in the MagRC chip. See supplementary information and Figures S1 - S3 for an explanation of the model and modeling data.

\section{Resolution, sensitivity, and versatility of the MagRC approach}

In a first suite of experiments, we used four cell lines having known levels of expression of the Epithelial Cell Adhesion Molecule (EpCAM) to challenge the capture and sorting capabilities of the MagRC chip. EpCAM is a surface marker commonly used totarget CTCs. It is known that CTCs lose EpCAM when they undergo the epithelial to mesenchymal transition (EMT) during cancer 
progression ${ }^{21,22}$, and therefore tracking this marker should allow EMT to be monitored. Four different target cell lines- MCF-7, SKBR-3 (breast adenocarcinoma cells), PC-3 (human prostate cancer cell line), and MDA-MB-231 (a breast cancer cell line with mesenchymal characteristics that mimics triple negative breast cancer cells) were incubated with $50 \mathrm{~nm}$ nanoparticles coated with anti-EpCAM in buffered solution. After capture, a nuclear stain was introduced into the chip to identify captured cells, and capture efficiency was assessed by counting the capture cells using fluorescent microscopy. Experiments for each cell line were repeated three times. Figure $3 \mathrm{~A}$ shows the fluorescent microscope images of an SKBR3 cell captured at the edge of a nickel micromagnet (where the magnetic field and field gradients are at a maximum).

The four different cell lines tested exhibited markedly different distributions within the device (Figures 3B and S5). High recoveries of the cells injected into the device are achieved (MCF-7 95 $\pm 5 \%$, SKBR3 93 $\pm 4 \%$, PC-3 91 $\pm 6 \%$, MDA-MB-231 $94 \pm 5$ ) (Figure $3 \mathrm{C}$ ), indicating that this approach has a high level of sensitivity. MCF-7 cells, which have the highest level of EpCAM expression, were found primarily in the earlier zones where the micromagnets are the smallest. However, PC-3 and MDA-MB-231 cells (which had the lowest level of EpCAM expression) were only captured after they encountered the large micromagnets closer to the outlet of the chip. The relative levels of EpCAM expression of the cell lines were confirmed via flow cytometry (Figure 3B inset). T-test analysis was used to assess statistical significance of MagRC profiles obtained from different cell lines (Table S1, S2 and S3). The calculated P-values $(<0.0001)$ confirm the statistical significance of the uniqueness of the MagRC profiles and that the resolution of this 


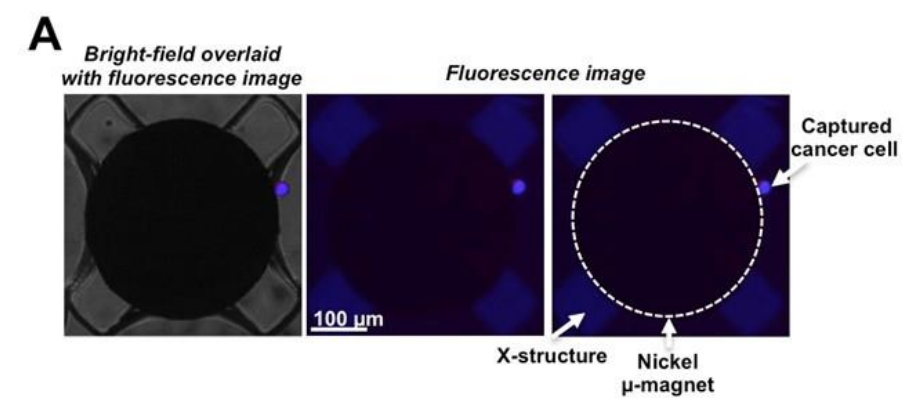

B

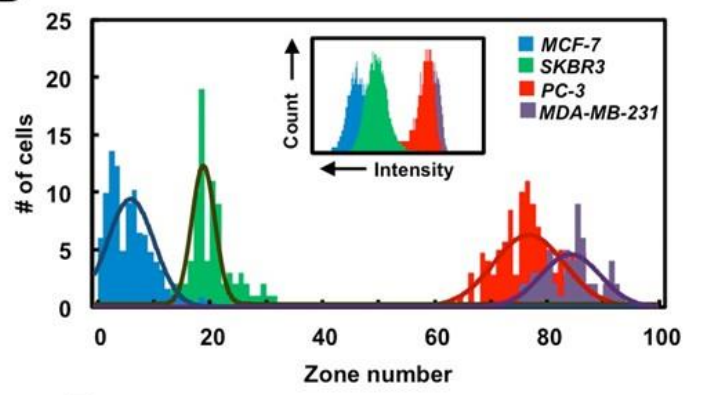

C

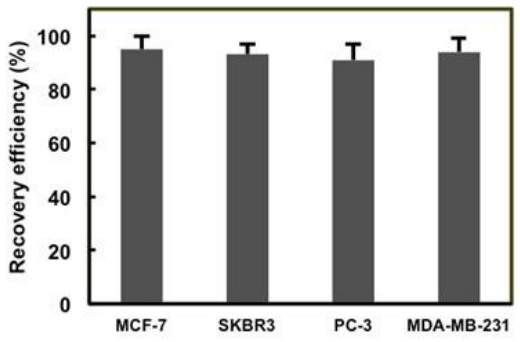

D

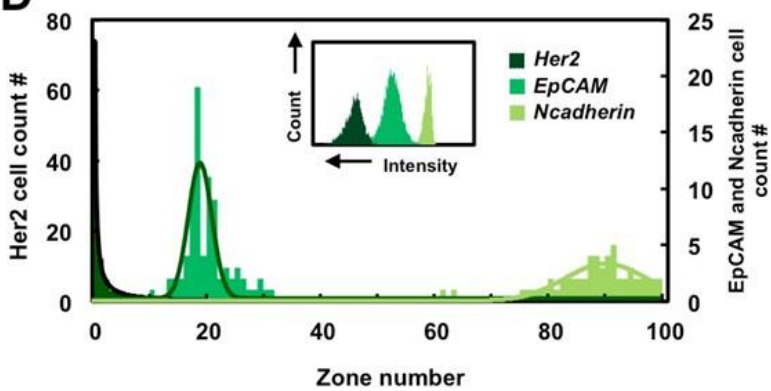

E

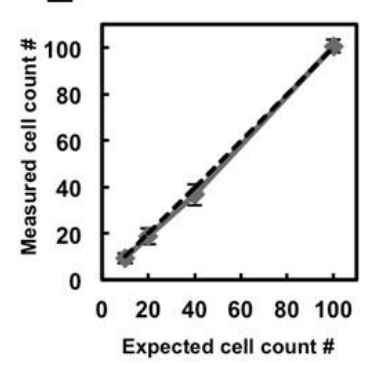

Fig. 3. Profiling protein surface expression using Magnetic Ranking Cytometry. A) Bright- field and fluorescent microscope images (left \& right, respectively) of a captured, immunostained SKBR3 cell. B) Distribution of VCaP, SKBR3, and MDA-MB-231 cells in the MagRC chip; EpCAM was used as the profiling marker. 100 cells suspended in $100 \mu \mathrm{l}$ of buffer were used in these trials. Profiling experiments for each cell line were repeated 5 times. Three replicates of capturing each cell line were shown in SI.The inset figure shows EpCAM expression measured by flow cytometry for the three cell lines. C) Capture efficiency for cells that have different levels of EpCAM expression. High recovery of low EpCAM cells (MDA-MB- 231 and PC-3) proves the suitability of the MagRC approach to monitor cells with lowered epithelial markers. D) SKBR3 cells were profiled for different cancer biomarkers using three capture antibodies: EpCAM, HER2, and N-Cadherin. 100 cells suspended in $100 \mu \mathrm{l}$ of buffer were used in these trials, and experiments were replicated three times. The inset figure shows expression of the same three markers on SKBR3 cells measured by flow cytometry. E) The sensitivity of the MagRC approach was tested by spiking different numbers of SKBR3 cells in buffer solution and counting them using immunofluorescence after capture in a MRC chip. A low number of cells $(n=10)$ spiked into a volume of $100 \mu$ can be visualized. Error bars show standard deviations, $n=3$. It is noteworthy that overlap can occur for the profiles collected from different cell lines, reflecting that surface expression levels for some cell subpopulations in different cell lines may be similar. As shown in the inset of Figure 3B, flow cytometry also generates overlapping profiles. 
technique is high. Based on these results we can conclude that the MagRC chip is able to sort cells according to the expression level of a targeted surface marker. Moreover, it efficiently captures cells exhibiting even low levels of a target surface marker, and can be applied widely to target surface markers for which a corresponding antibody is available.

This magnetic ranking cytometry approach is amenable to the use of a wide range of surface antigens as the basis for profiling. We profiled the SKBR3 cell line using three different surface markers that are often expressed in epithelial cancer cells: human epidermal growth factor receptor 2 (HER2)/neu, EpCAM, and $\mathrm{N}$-cadherin (Figure 3D). The inset in Figure 3D shows the level of these three surface markers in SKBR3 cells measured by flow cytometry. HER2 is known to be highly overexpressed in this cell line, and indeed, experiments with magnetic nanoparticles coated with anti-HER2 led to cell capture within the very earliest zones of the chip. In contrast, capture with anti-N-cadherin coated nanoparticles showed most cells being captured in the later zones of the chip. EpCAM levels are intermediate for these cells, a fact also reflected in the MagRC profile.

The data presented indicate that magnetic ranking cytometry produces profiles comparable to those reported by flow cytometry (FCM). FCM is a powerful and robust approach useful in analyzing protein expression and heterogeneity in living cells. It is limited in its sensitivity, however, and requires cell numbers of $10^{4}$ or higher for accurate results ${ }^{23}$. As shown here, MagRC reports on protein expression with similar resolution, but using much smaller collections of cells. It is also noteworthy that the MagRC approach is a gentle analysis method that allow high recoveries of viable cells. $92 \%$ of captured cells can be recovered, and $98 \%$ of the recovered cells are viable (Figure S7). 
We then proceeded to challenge the system using unprocessed whole blood samples, and found that MagRC retains its sensitivity and profiling capability. When whole blood samples $(1 \mathrm{ml})$ containing between 10 and 40 cells were profiled using EpCAM as a target marker, reproducible profiles were obtained (Figure 4). We compared the performance of the MagRC approach with the CTC gold standard FDA-cleared CellSearch assay (Figure 4B). Spiked blood samples containing 100 SKBR3, PC-3 and MDA-MB-231 cells per milliliter were prepared and analyzed using both MagRC chip and CellSearch. High recoveries of the spiked samples injected into the MagRC chip were achieved (SKBR3 97 $\pm 3 \%$, PC-3 $90 \pm 2 \%$, MDA-MB-231 $90 \pm 3 \%$ ). The efficient capture of MDA-MB-231 and PC-3 cells, which have a low level of EpCAM, indicates that low EpCAM cells presented in whole blood sample would still be visualized with this approach. However, in contrast, the CellSearch system exhibits significantly suppressed capture efficiencies with low EpCAM cells.

To validate further the phenotypic profiling performance of the MagRC approach in whole blood, we performed head-to-head studies of blood samples containing 100 cancer cells where both MagRC and FCM were used for profiling. MagRC profiled cells in the presence of normal blood cells (Figure 4C, 4D, and $4 \mathrm{E}$ ), while FCM was unable to report a specific signal (Figure 4E). Even in the presence of 10,000 cells spiked into blood, a specific signal was not obtained using FCM. Only after the blood was treated to lyse red blood cells could spiked cancer cells be visualized; unfortunately, this processing step eliminates over $50 \%$ of the cancer cells (Figure S8), and therefore creates the significant potential for false negatives. In contrast with FCM, the MagRC approach provides accurate profiling even with very low levels of cancer cells in unprocessed blood. This is a requirement for the evaluation of CTCs. It is noteworthy that the exact shape of the profile returned with MagRC is affected by the presence of blood cells (Figure 4E); however, since it is affected in a consistent and predictable fashion by the increased drag acting on the tumour cells that arises from interactions with the blood cells, it gives reproducible data for a given type of sample (e.g. whole blood). 
The purity of cancer cells recovered during MagRC profiling was assessed by counting the numbers of WBCs non-specifically captured within our devices. The MagRC chip depletes up to $99.98 \%$ of the WBCs, with approximately 2000 WBCs found in the chip after processing $1 \mathrm{ml}$ of blood. While much of this contamination is derived from non-specific binding of WBCs to the device, we wondered if non-specific binding of magnetic beads could also contribute to the capture of these cells. We used flow cytometry to compare the specific binding of particles to MDA-MB-231 cells and the non-specific binding to WBCs (Figure S4). The data from this experiment indicated that the level of non-specific binding of the magnetic nanoparticles to WBCs is 10x lower than that occurring on low EpCAM cells, indicating that WBCs would not be captured within our device via this mechanism. The level of WBC contamination found in MagRC chip is comparable to other microfluidic capture approaches, including the micropost CTC chip with $640 \mathrm{WBCs}$ isolated per $1 \mathrm{~mL}$ of blood $^{6}$, the microvortex-generating herringbonechip with $4500 \mathrm{WBCs} / \mathrm{mL}^{9}$, and the tunable nanostructured coating approach with $1200 \mathrm{WBCs} / \mathrm{mL}^{14}$. For our approach, along with the others described where a positive selection approach is used, the ability to identify cancer cells specifically using immunofluorescence ensures that these non-specifically bound cells do not contribute to the results obtained. 
B
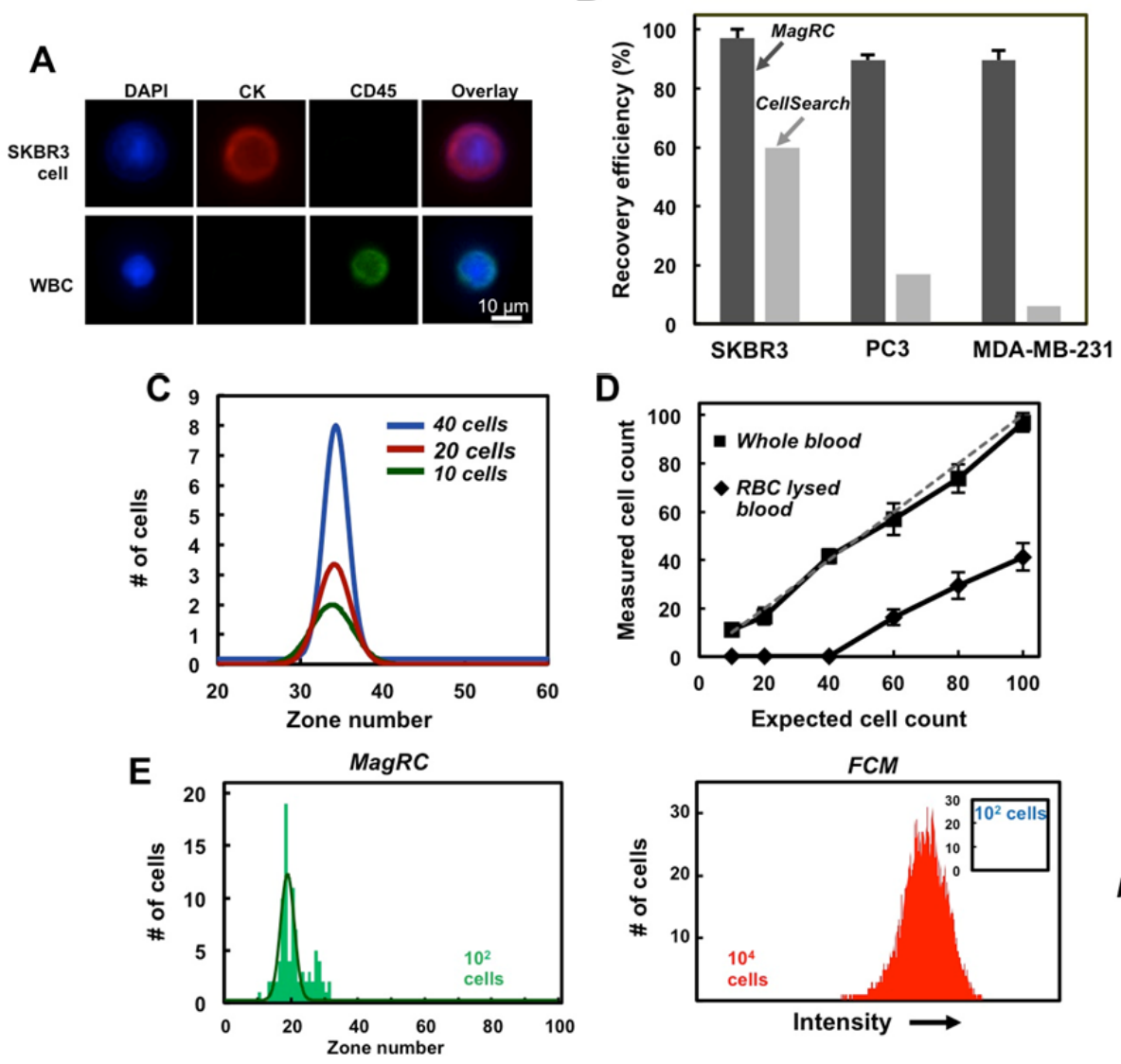

PBS
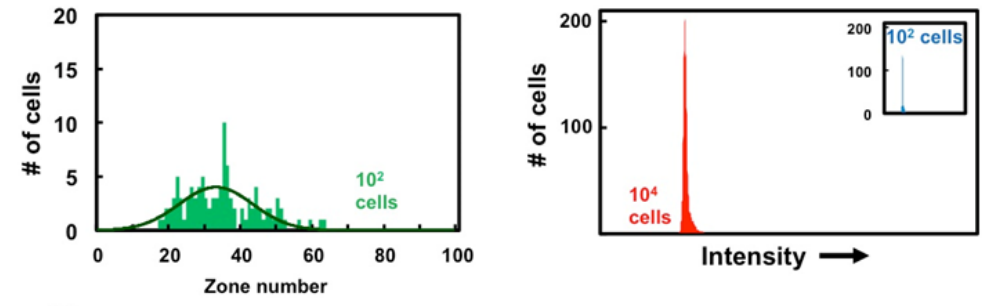

Whole
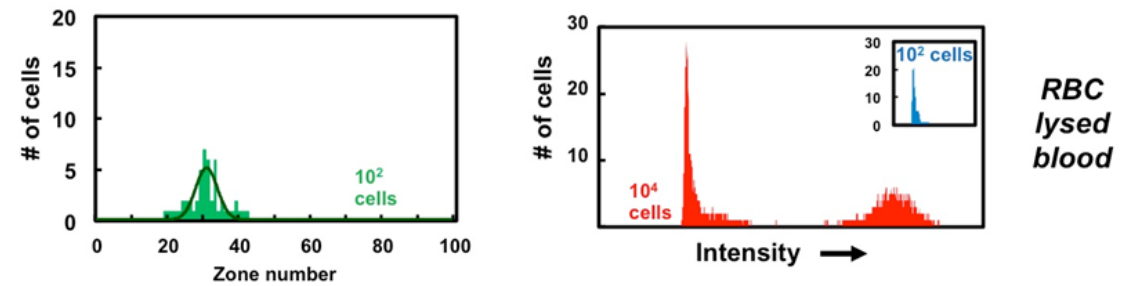

Fig. 4. Magnetic Ranking Cytometry applied to rare cells in whole blood. A) Specific immunostaining of cancer cells. After capture, cancer cells are stained for DAPI, CK, and CD45. SKBR3 cells were identified as $\mathrm{DAPI} / \mathrm{CK}^{+} / \mathrm{CD} 45^{-}$and white blood cells were identified as $\mathrm{DAPI} / \mathrm{CK}^{-} / \mathrm{CD}^{+} 5^{+}$. B) Head-to-head comparison of the MagRC chip with CellSearch. 100 cells of SKBR3, PC3 and MDA-MB-231 cells were spiked into whole blood. MagRC and CellSearch were used to count cells. CellSearch was inefficient to recover low EpCAM cells while MagRC retains cells with efficiency more than $90 \%$. C) Different numbers of SKBR3 cells were spiked in $1 \mathrm{ml}$ of whole blood and the MagRC chip was used to profile the spiked samples for surface expression of EpCAM. Experiments were repeated three times. D) MagRC was used to count rare cells in unprocessed whole blood samples and RBC-lysed samples. A significant proportion of cells are lost when this sample processing step is used. In unprocessed blood, MagRC shows high levels of sensitivity and linearity. See Figure S8 for raw data. Cells were spiked into $1 \mathrm{ml}$ of human blood for all trials shown. Error bars show standard deviations, $n=3$. E) Both flow cytometry and the MagRC chip were used to monitor cells in PBS, whole blood, and RBC-lysed blood. The MagRC chip was able to accurately profile cells accurately in all three solutions. However, the background signal for whole blood samples overwhelmed the signals collected via FCM; only cells in PBS and RBC-lysed blood samples were accurately measured using the technique. Due to the inability of FCM to accurately count low ( 100) numbers of spiked cells (inset), samples with a higher level of SKBR3 cells $\left(10^{4}\right)$ were measured and counted using FCM. Profiling with both FCM and MagRC was repeated three times. 


\section{Monitoring dynamic CTC phenotypes in an animal model of cancer}

To evaluate the utility of magnetic ranking cytometry for the analysis of CTCs and their dynamic properties, we first analyzed blood from mice bearing xenografted tumours as a function of tumour growth. To generate the model, we implanted MCF-7/Luc human breast cancer cells into the mammary fat pad of immunodeficient mice. One group of mice received an estrogen pellet prior to tumour implantation $\left(E_{+}\right)$, as estrogen stimulates MCF-7 tumour growth. Mice in the other set were not treated with estrogen prior to tumour implantation. After tumour cell injection, we collected blood from each mouse every 10 days and analyzed the samples using MagRC. Immunostaining that was specific for the implanted human cancer cells was used to establish the MagRC profile (Figure $5 \mathrm{~A})$, and tumour growth was visualized by imaging the bioluminescence generated by the luciferin-tagged MCF-7 cells (Figure 5B).

As tumour growth progressed in the xenografted animals, a marked change was visualized in the CTCs detected. In both the estrogen-positive and -negative animal groups, CTC levels rose as the study progressed. In the estrogen-positive group, as expected, the CTCs levels increased to a much higher level than in the estrogen-negative group. Notably, in addition to increasing in number, the more aggressive cancer model also exhibited a marked phenotypic shift: the CTCs profiled in these mice were localized in later zones within the MagRC microfluidic chip compared to early CTCs and cultured MCF-7 cells (Figure 3B and Figure S9). The profiles indicate that the phenotypes of the CTCs were changing and EpCAM levels were decreasing (Figure 5C, S10, and S11). The profiles of the CTCs from the estrogen-negative mice remained static (Figure 5D, S10, andS11). 

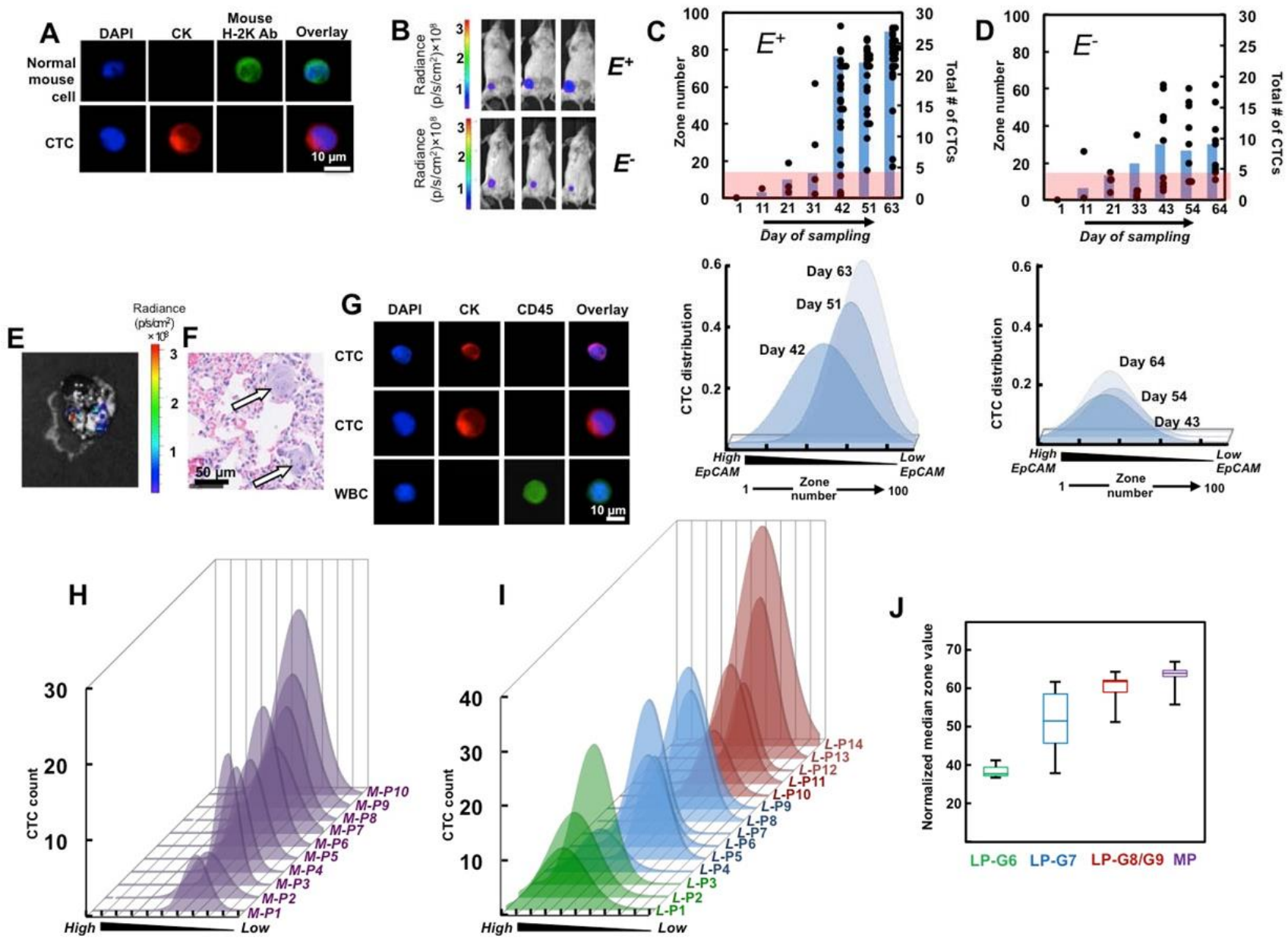

J

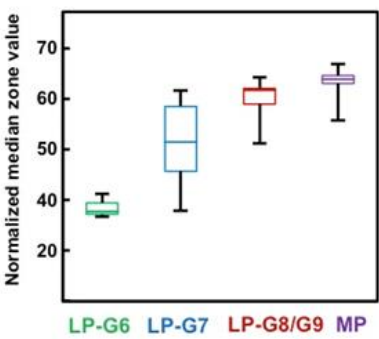

Fig. 5. MagRC enables profiling of CTCs in cancer xenograft models and patient samples. A) Representative images of a captured CTC and a normal mouse cell. Nuclei are stained with DAPI (blue), CTCs are stained for CK (red), and mouse cells for mouse H-2k (green). B) Bioluminescence images of mice implanted with MCF-7 tumours in estrogen positive $\left(E^{+}\right)$and estrogen negative ( $\left.E^{-}\right)$ groups during the course of tumour progression. C \& D) CTC distribution profiles of mice in $E^{+}$and $E^{-}$groups. Bar graphs show the total number of CTCs found in each day. Each black circle denotes one CTC. The red zone represents the distribution area for cultured MCF-7 cells (See Fig. S9). Scaled normal distribution profiles of CTCs extracted at each time point are shown at the right side of the panel, centered at the median CTC zonal position. CTC profiles in the $\mathrm{E}^{+}$model show a shift toward less epithelial phenotypes at the later stages of the disease $(\mathrm{C})$, however, this shift is not observed in E model (D). See Fig. S10 and S11 for additional data collected with animals. Since our sample size (the number of mice) is less than 5, we have not used any statistical analysis, and individual data points were plotted. E) Bioluminescence image of whole lung of a mouse in the E+ group. Visible luminescence indicates the presence of metastases in lung. F) Histopathology image of lung section of a mouse from the $\mathrm{E}+$ group confirming the presence of micrometastases. G) Representative images of CTCs captured from prostate cancer patient samples versus a white blood cell. Nuclei are stained with DAPI (blue), CTCs are stained for CK (red), and WBCs for CD45 (green). H) EpCAM profiles for CTCs captured from samples from patients with metastatic, castrationresistant prostate cancer $(n=10)$. See supplementary table S6 for patient data. I) EpCAM profiles for CTCs captured from samples from patients with localized prostate cancer $(n=14)$. See supplementary table S5 for patient data. Patients with tumours with Gleason score of 6 are colored green (P1-P3), Gleason score 7 are blue (P4-P9), and Gleason score 8 and 9 are red (P10-P14). See Figure S12 for mean capture zone values and Tale $S 7$ for statistical analysis of these values. J) Box plot indicating median values corresponding to capture profiles for localized prostate cancer patients with Gleason score 6 tumors (LP-G6), Gleason score 7 tumors (LP-G7), Gleason score 8 or 9 tumors (LPG8/G9), or metastatic castrate-resistant prostate cancer patients (MP). 
To compare invasiveness of the tumours in the two groups, we extracted mouse lungs and sent them for histopathology at the end of the study, where the sections were scanned for micrometastases. Micrometastases were found in lungs of the $\mathrm{E}+$ group (Figure $5 \mathrm{E}$ and $5 \mathrm{~F}$ ); and there were no micrometastases in the $\mathrm{E}^{-}$ group. The presence of the metastases along with the altered CTC profile observed by MagRC is consistent with the hypothesis that the CTCs produced by the estrogen-positive tumour possess a more invasive profile.

\section{Profiling phenotypes of CTCs in clinical samples}

To evaluate the performance of MagRC when tested with clinical samples, we conducted a study of samples collected from patients exhibiting metastatic castration-resistant prostate cancer ( $m C R P C, n=10)$ and localized prostate cancer, ( $n=14$, Figure $5 \mathrm{G}, 5 \mathrm{H}, 5 \mathrm{I}$ and Table $\mathrm{S} 5$ \& S6). Immunostaining was used to distinguish between CTCs and WBCs (Figure 5G). We also analyzed the blood collected from 9 healthy donors (Table S4).

The profiles collected from the different patients exhibited an interesting series of trends (Figure 5). O Overall, the MagRC profiles for the MCRPC patients were similar to one another (Figure $5 \mathrm{H}$ ). The CTCs from these patients appeared in the later zones of the device, consistent with the idea that these were lowEpCAM CTCs in later stages of EMT. In the case of localized prostate cancer patients, there was an appreciably greater diversity in the MagRC profiles (Figure 5I). We analyzed the profiles according to the Gleason score of the tumours biopsied in these patients. Three, six, and five patients with tumours with Gleason score of 6 (P1-P3), Gleason score 7 (P4-P9), and Gleason scores 8 and 9 (P10P14) were analyzed, respectively. We measured the zone distribution profile for these patients, and found that G6 patient CTCs were captured in earlier zones (median zone $=40$ ) relative to the CTCs captured from samples from patient with G8/G9 tumours (median zone $=64$ ) (Figure S12). The boxplot presented in Figure

$5 \mathrm{~J}$ also demonstrates the CTC profile distribution in patients with different types of prostate cancer tumours. These results suggest that the patients with G7 tumours exhibited variable profiles compared to the other two groups. We performed 
statistical analysis on the localized prostate cancer patient CTC zone distributions, and found that G8-G9 CTCs were statistically separated from G6 CTCs (Table S7, $\mathrm{P}<0.05$, paired t-test). The $\mathrm{G} 7$ tumour profile mean values did not exhibit statistical significance from the $G 6$ or $G 8 / G 9$ patients, indicating significant phenotypic heterogeneity for the $G 7$ patients. This is an interesting finding because G7 patients have variable prognoses; while $50 \%$ of patients with $\mathrm{G} 7$ tumours do experience cancer recurrence, $50 \%$ do not ${ }^{24}$. A much larger study will be necessary to determine whether there is a correlation between the CTC phenotypic profiles we are measuring and recurrence, but the analysis of CTC phenotypes for these patients may help elucidate the differences between tumours with similar staging data.

\section{CONCLUSIONS}

The high level of sensitivity we report for phenotypic profiling of CTCs using MagRC, and the device's efficacy in the analysis of whole blood renders this a technique of interest in the analysis of rare circulating tumour cells. CTCs collected from mice with xenografted tumours were monitored as a function of tumour growth, and dynamic phenotypic profiles were observed for cells collected from animals with aggressive tumours. In samples collected from prostate cancer patients, MagRC enabled the sensitive profiling of CTCs and monitoring of changes in CTC levels and phenotypes. A comparison of CTCs profiled in samples collected from patients with localized versus metastatic prostate cancer revealed that there was much greater diversity in the phenotypes of CTCs for the former group versus the latter.

The MagRC approach stands to be adapted to a variety of applications. Because an external magnetic field modulates CTC capture, cells can readily be recovered once the field is removed, thereby facilitating further offline analysis and culture. This technique is highly versatile inasmuch as it can employ available antibodies to generate a MagRC profile, which makes it applicable to CTC analysis relevant to a variety of disease states. The new technique can be implemented using standard syringe pumps and fluorescence imaging interfaced with a 
microfluidic chip that is straightforward to fabricate; without the requirement of custom instrumentation. Further effort at system integration will permit deployment of this technology in clinical research and clinical cancer management.

Magnetic ranking cytometry is the first technique to provide accurate in-line profiles of low levels of CTCs in unprocessed blood samples. While techniques developed previously have leveraged surface-bound magnetic particles for CTC enumeration ${ }^{13,25}$, none has achieved the level of sensitivity and resolution that MagRC exhibits. Further, none have provided the ability to report a protein expression profile for CTCs. MagRC provides information consistent with that provided by the existing gold standard method, flow cytometry, and, further, allows vastly lower cell numbers to be queried. Further, the acquisition of sensitive information using MagRC is not degraded by the presence of an abundance of blood cells, thereby overcoming a significant limitation of flow cytometry.

\section{METHODS}

MagRC microfluidic chip fabrication. Glass slides coated with $1.5 \mu \mathrm{m} \quad \mathrm{Ni}$ layer (EMF-Corp, Ithaca, NY) were used to fabricate the MagRC chip. A top-coat of positive photoresist (AZ1600) was used. Contact lithography was used to pattern the micro-magnets. After exposing for 10 seconds, photoresist was developed. This was followed by Ni wet etching and removal of the top resist. To pattern the ' $X$ '-structures, a layer of negative photoresist, SU-8 3050 (Microchem, Newton, MA) was spin-coated on top of the nickel coated glass substrates followed by 30 minutes soft-baking. The final thickness of SU-8, and thus the height of channel, was $50 \mu \mathrm{m}$. After exposing for 20 seconds, the SU-8 layer was developed using SU-8 developer. Lastly, the channel was topped with a layer of polydimethylsiloxane (PDMS). In PDMS layer, holes were punched as inlet and outlet.

Cancer cell lines. MCF-7/Luc human breast cancer cells were purchased from Cell Biolabs Inc. and grown in DMEM (High Glucose) supplemented with 10\% FBS, $0.1 \mathrm{mM}$ MEM Non-Essential Amino Acids (NEAA) and $2 \mathrm{mM}$ L-glutamine. SKBR3 cell line was obtained from American Type Culture Collection (ATCC). 
SKBR3 cells were cultured in McCoy's 5a Medium Modified (ATCC). The media was supplemented with $10 \%$ fetal bovine serum (FBS). Human prostate cancer cells PC-3 (a kind gift from Dr. Alison Allan, London Health Sciences Centre, London, ON.) were cultured in F12K media (ATCC) supplemented with $10 \%$ FBS. All cell lines were authenticated using gene expression profiling and checked for microbial contaminations.

Assessing the level of magnetic beads adsorption to WBC. Fresh blood was collected from healthy donors and RBCs were lysed using 0.5M EDTA, pH 8 . MDA-MB-231 and SKBR3 cells also were prepared with the concentration of $10^{5}$ cells $/ \mathrm{mL}$ in PBS plus 1\%BSA. Then 10 $\mu$ l of anti-EpCAM Nano-Beads (MACS) and $20 \mu \mathrm{l}$ of FcR Blocking Reagent (MACS) were added to the samples (MDA-MB-231 cells, SKBR3 cells and RBC-lysed blood) and incubated for 30 minutes. After washing step, the samples were incubated for another 30 minutes with $1.5 \mu \mathrm{L}$ of Anti-mouse H-2Kd-Alexa Fluor 488 that served as the secondary antibody. It was followed by injection of samples into the flow cytometer. Counts versus fluorescence intensity measurements were made in the green channel of the flow cytometer.

Spiking of tumour cells in whole blood. Fresh blood was collected from healthy volunteers, and immediately used for experiments. Different numbers of SKBR3 cells were spiked into whole blood. After this step, some samples underwent an additional RBC lysis step; $1 \mathrm{~mL}$ of RBC lysis buffer was used, and this was followed by two washing steps with PBS. Lastly, both whole and RBClysed blood samples were run through the MagRC chip and analysed via flow cytometry.

Orthotropic tumour xenograft model and CT imaging. All animal experiments were carried out in accordance with the protocol approved by the University of Toronto Animal Care Committee. 6- to 8-week-old female SCID-beige mice were purchased from Charles River and maintained at the University of Toronto animal facility. 2 days prior to tumour implantation, the subset of mice received a subcutaneous pellet of $60-d$ sustained release $17-\beta$-estradiol $(0.72$ mg/pellet; Innovative Research of America). Tumour xenografts were generated by 
injecting $5 \times 10^{6}$ cells suspended in $50 \mu$ of Matrigel (BD Biosciences) orthotopically into the 4th left inguinal mammary fat pad. Mice were anaesthetized by isoflurane before injection. Tumour growth was measured both by caliper and by imaging using a Xenogen IVIS Spectrum imaging system (Caliper Life Sciences). If tumour growth was not observed in a mouse, it was excluded from the rest of study. Prior to imaging, mice were injected intraperitoneally with $100 \mu$ of phosphate-buffered saline containing D-Luciferin substrate (PerkinElmer). At the end of the experiment, animals were euthanized and selected tissues were analyzed by ex-vivo imaging for micro-metastasis detection. For intermediate CTC capture from tumour bearing mice, $50-100 \mu \mathrm{l}$ of blood was collected from the saphenous vein and for the terminal studies $0.5 \mathrm{ml}-1 \mathrm{ml}$ blood was collected from each mouse by cardiac puncture. All blood samples were collected in K2EDTA tubes (Microvette, Sarstedt). During the animal study, randomization was notapplied.

Profiling of mouse CTCs and immunostaining. Collected mouse blood was diluted with PBS-EDTA (100 $\mu \mathrm{L}$ of PBS-EDTA was added to $50 \mu \mathrm{L}$ of blood). This was followed by adding $10 \mu \mathrm{L}$ of anti-EpCAM Nano-Beads to $150 \mu \mathrm{L}$ of diluted blood. After 30 minutes incubation with the magnetic beads, blood was pumped through the MagRC Chip at a flow rate of $500 \mu \mathrm{L} / \mathrm{h}$. Next, $200 \mu \mathrm{L}$ PBS-EDTA was introduced to flush away any non-magnetically captured non-target cells. Captured cells were then fixed with $4 \%$ paraformaldehyde, and then permeabilized with $0.2 \%$ Triton X-100 (Sigma-Aldrich) in PBS. Anti-CK-APC (GeneTex) antibody was used to stain CTCs, and mouse cells were marked by anti-mouse-H-2K-FITC antibody to distinguish with CTCs. All antibodies were prepared in $100 \mu \mathrm{L}$ of PBS and pumped through the chip at a flow rate of $50 \mu \mathrm{L} / \mathrm{hr}$ for 2 hrs. After immunostaining, chips were washed using $0.1 \%$ Tween 20 in PBS. Cell nuclei were stained with $100 \mu \mathrm{l}$ DAPI ProLong Gold reagent (Invitrogen, CA) at $500 \mu \mathrm{L} / \mathrm{h}$. After completion of staining, all chips were washed with PBS and stored at $4{ }^{\circ} \mathrm{C}$ before scanning.

Image scanning and analysis. After immunostaining, chips were scanned using a Nikon microscope under 10X objective, and images were acquired with NIS-Elements AR software. Bright field, red (APC channel), green (FITC channel) 
and blue fluorescence images were recorded. The captured images were then analyzed manually and target and non-target cells were counted.

Histopathology of mouse tumours. After terminal blood collection, animals were euthanized and lungs, liver, lymph nodes were extracted and fixed in $10 \%$ buffered formalin. Fixed tissues were then embedded in paraffin for histological examination with hematoxylin and eosin (H\&E) staining. The pathologist was blinded to the different groups of animals during the study.

Patient sample collection. Metastatic castration-resistant prostate cancer patients were recruited from the Princess Margaret Hospital according to the University's Research Ethics Board approved protocol, and localized prostate cancer patients were recruited from Sunnybrook Hospital under an approved protocol. All patients were enrolled following informed consent. $20 \mathrm{ml}$ peripheral blood samples from castration-resistant prostate cancer patients $(n=10)$ were used to validate CTC detection with magnetic ranking cytometry versus CellSearch. Blood samples were collected in two CellSearch tubes containing the anticoagulant EDTA (Johnson and Johnson). One tube of blood was shipped to the London Regional Cancer Program at the London Health Sciences Centre for CellSearch analysis, and the other tube of blood was processed using magnetic ranking cytometry. $10 \mathrm{ml}$ peripheral blood samples from localized prostate cancer patients $(n=14)$ were also analyzed using magnetic ranking cytometry. All samples were analyzed within a 96-hour window, typically within 24- 48 hours after blood collection. Blood from 10 healthy donors was also analyzed for comparison.

CTC capture from patient samples. $10 \mu \mathrm{l}$ of anti-EpCAM Nano-Beads (MACS) and $20 \mu \mathrm{l}$ of FcR Blocking Reagent (MACS) were added to $1 \mathrm{ml}$ of blood and incubated for 30 minutes on a sample mixer. The blood was then introduced into a microfluidic device based on a simplified design24 at a flow rate of $600 \mu \mathrm{l} / \mathrm{h}$ using a syringe pump. Next, $400 \mu \mathrm{l}$ of PBS-EDTA was added at a flow rate of $600 \mu \mathrm{l} / \mathrm{h}$ to remove non-target cells. After this step, the chips were immunostained as detailed below.

Immunostaining of patient CTCs. After processing the blood, cells were fixed with $4 \%$ paraformaldehyde, and subsequently permeabilized with $0.2 \%$ Triton 
X-100 (Sigma-Aldrich) in PBS. Cells were immunostained with primary antibodies, biotin-Mouse monoclonal Cytokeratin 18 (Lifespan), and mouse monoclonal CD45APC (ThermoFisher), followed by Yellow-Avidin nanobeads (Invitrogen) $(1: 2500)$ to visualize the CTCs. All of the antibodies were prepared in $100 \mu \mathrm{l}$ PBS plus $1 \%$ BSA and $0.1 \%$ Tween20 and chips were stained for 60 minutes at a flow rate of $100 \mu \mathrm{l} / \mathrm{h}$. Chips were washed using $200 \mu \mathrm{l} 0.1 \%$ Triton X-100 in PBS, at $0.6 \mathrm{ml} / \mathrm{h}$ for $10 \mathrm{~min}$. Chips were stained with the Yellow-Avidin nanobeads for 30 minutes at a flow rate of $200 \mu \mathrm{l} / \mathrm{h}$, and subsequently washed with $200 \mu \mathrm{l}$ of $0.1 \%$ Triton X-100 in PBS, at $600 \mu \mathrm{l} / \mathrm{h}$ for $10 \mathrm{~min}$. Nuclei were stained with $100 \mu \mathrm{l}$ DAPI ProLong Gold reagent (Invitrogen, CA) at $600 \mu \mathrm{l} / \mathrm{h}$. After completion of staining, all devices were washed with PBS and stored at $4^{\circ} \mathrm{C}$ before scanning.

Antibodies. The following antibodies were used in this study: CD326 (EpCAM) MicroBeads (130-061-101, MACS-dextran ferrite colloids beads with a diameter of $50 \mathrm{~nm}$, purchased from Miltenyi Biotec), pan-Cytokeratin antibody [C11] -APC (GTX80205); CD45 Mouse Anti-Human mAb (clone HI30)-Alexa Fluor 488 (MHCD4520); anti-human CD326 (EpCAM)-Alexa Fluor 647 (324212); Antimouse H-2Kd- Alexa Fluor 488 (116609); CK-18 mouse anti-human antibody clone C-04, Biotin (LS-B9908), CD45-APC mouse anti-human clone HI30 (MHCD4505), Yellow- Avidin nanobeads (F8771), DAPI nuclear stain (R37605), FcR Blocking Reagent (130-059-901).

ACKNOWLEDGEMENTS. The authors acknowledge generous support from the Canadian Institutes of Health Research (Emerging Team grant, POP grant), the Ontario Research Fund (ORF Research Excellence grant), the Canadian Cancer Society Research Institute (Innovation grant), and the Connaught Foundation. We also gratefully acknowledge all of the patients and healthy donors who donated specimens to our studies.

Author contributions. M.P., P.A., S.A. B.G., L.K., V.N., C.T., R.M., S.O.K. and E.H.S. conceived and designed the experiments: M.P., P.A., S.A. B.G., L.K., V.N., C.T., R.M., performed the experiments and analyzed the data: R.K., A.H., S. S., A.F., N.F. and A.J. contributed clinical expertise and clinical specimens. All 
authors discussed the results, and contributed to the preparation and editing of the manuscript.

Competing financial interest statement. The authors do not have any competing financial interests.

Supplementary information available. Supplementary information is available in the online version of the paper. Reprints and permission information is available online at www.nature.com/reprints. Correspondence and requests for materials should be addressed to S.O.K. and E.H.S.

\section{REFERENCES}

(1) Chaffer, C. L., Weinberg, R. A. A perspective on cancer cell metastasis. Science 331, 1559-1564 (2011).

(2) Plaks, V., Koopman, C. D., Werb, Z. Circulating tumour cells. Science 341, 1186-1188 (2013).

(3) Alix-Panabieres, C., Pantel, K. Challenges in circulating tumour cell research. Nat. Rev. Cancer 14, 623-631 (2014).

(4) Lang, J. M., Casavant, B. P., Beebe, D. J. Circulating tumour cells : getting more from less. Sci. Transl. Med. 4, 1-5 (2012).

(5) Green, B., Saberi Safaei, T., Mepham, A., Labib, M., Mohamadi, R.M., Kelley, S.O. Beyond CTC capture: next-generation devices and materials. Angew. Chem. Intl. Ed. 55, 1252-1265 (2016).

(6) Hu, X.; Bessette, P. H.; Qian, J.; Meinhart, C. D.; Daugherty, P. S.; Soh, H. T. Marker-specific sorting of rare cells Using dielectrophoresis. Proc. Natl. Acad. Sci., U.S.A. 15, 15757-15761 (2005).

(7) Nagrath, S., Sequist, L. V, Maheswaran, S., Bell, D. W., Irimia, D., Ulkus, L., Smith, M. R., Kwak, E. L., Digumarthy, S., Muzikansky, A., Ryan, P., Balis, U. J., Tompkins, R. G., Haber, D. A., Toner, M. Isolation of rare circulating tumour cells in cancer patients by microchip technology. Nature, 450, 12351239 (2007).

(8) Adams, A., Okagbare, P. I., Feng, J., Hupert, M. L., Patterson, D., Go, J. Highly efficient circulating tumour cell isolation from whole blood and label- 
free enumeration using polymer-based microfluidics with an integrated conductivity sensor. J. Am. Chem. Soc. 130, 8633-8641 (2008).

(9) Talasaz, A. H. et al. Isolating highly enriched populations of circulating epithelial cells and other rare cells from blood using a magnetic sweeper device. Proc. Natl. Acad. Sci., U.S.A. 106, 3970-3975 (2009).

(10) Stott, S. L. et al. Isolation of circulating tumour cells using a microvortexgenerating herringbone-chip. Proc. Natl. Acad. Sci., U.S.A. 107, 1839218397 (2010).

(11) Wang, S. et al. Highly efficient capture of circulating tumour cells by using nanostructured silicon substrates with integrated chaotic micromixers. Angew. Chem. Intl. Ed. 50, 3084-3088 (2011).

(12) Schiro, P. G., Zhao, M., Kuo, J. S., Koehler, K. M., Sabath, D. E., Chiu, D. T. Sensitive and high-throughput isolation of rare cells from peripheral blood with ensemble-decision aliquot ranking. Angew. Chem. Intl. Ed. 51, 46184622 (2013).

(13) Zhao, W. et al. Bioinspired multivalent dna network for capture and release of cells. Proc. Natl. Acad. Sci., U.S.A. 109, 19626-19631 (2012).

(14) Ozkumur, E. et al. Inertial focusing for tumour antigen-dependent and independent sorting of rare circulating tumour cells. Sci. Transl. Med 5, 179ra47 (2013).

(15) Reátegui, E. et al. Tunable nanostructured coating for the capture and selective release of viable circulating tumour cells. Adv. Mater. 27, 15931599 (2015).

(16) Mittal, S., Wong, I. Y., Yanik, A. A., Deen, W. M., Toner, M. Discontinuous nanoporous membranes reduce non-specific fouling for immunoaffinity cell capture. Small 9, 4207-4214 (2013).

(17) Schneider, J., Bachmann, T., Franco, D., Richner, P., Galliker, P., Tiwari, M. K., Ferrari, A., Poulikakos, D. A novel 3D integrated platform for the highresolution study of cell migration plasticity. Macromol. Biosci. 13,973-983 (2013).

(18) Mohamadi, R. M. et al. Nanoparticle-mediated binning and profiling of heterogeneous circulating tumour cell subpopulations. Angew. Chem. Intl. Ed. Engl. 54, 139-143 (2015).

(19) Ferguson, B.S. et al. Genetic analysis of H1N1 influenza virus from throat swab samples in a microfluidic system for point-of-care diagnostics. J. Am. 
Chem. Soc., 133, 9129 (2011).

(20) Chen, P., Huang, Y.-Y., Hoshino, K., Zhang, J. X. J. Microscale magnetic field modulation for enhanced capture and distribution of rare circulating tumour cells. Sci. Rep. 5, 8745 (2015).

(21) Santisteban, M. et al. Immune-induced epithelial to mesenchymal transition in vivo generates breast cancer stem cells. Cancer Research, 69, 28872895 (2009).

(22) $\mathrm{Yu}, \mathrm{M}$. et al. Circulating breast tumour cells exhibit dynamic changes in epithelial and mesenchymal composition. Science 339, 580-584 (2013).

(23) Jaye, D. L., Bray, R. A., Gebel, H. M., Harris, W.A.C., Waller, E. K. Translational applications of flow cytometry in clinical practice. J. Immunol. 188, 4715-4719 (2012).

(24) Chan, T.Y.; Partin, A.W.; Walsh, P.C.; Epstein, J.I. Prognostic significance of gleason score $3+4$ versus gleason score $4+3$ tumour at radical prostatectomy. Urology, 56, 823-827 (2000).

(25) Issadore, D. et al. Ultrasensitive clinical enumeration of rare cells ex vivo using a micro-hall detector. Sci. Transl. Med. 4, 141ra192 (2012). 